\title{
A study on nabla discrete fractional operator in mass - spring - damper system
}

\author{
Okkes Ozturk \\ Department of Mathematics, Faculty of Arts and Sciences, Bitlis Eren University, Bitlis Turkey
}

Received: 14 April 2016, Accepted: 7 October 2016

Published online: 29 October 2016.

\begin{abstract}
The fractional calculus that is one of the new trends in science and engineering is concept of derivative and integral with arbitrary order. And, discrete fractional calculus (DFC) has an important place in fractional calculus which studied for the last 300 years. In present paper, we solved the equations of motion in mass-spring-damper system by using nabla $(\nabla)$ discrete fractional operator. And, we also introduced some instructive examples.
\end{abstract}

Keywords: Fractional calculus, discrete fractional calculus, nabla discrete fractional operator, mass-spring-damper system.

\section{Introduction}

Derivative and integral operators are important subjects in ordinary calculus. Similarly, sum and difference operators are also important subjects in discrete calculus. These operators can be applied to a function up to the $n$-th order where $n$ is an integer. However, in the fractional calculus, derivative or integral operators have arbitrary orders. It is well known that there is a similarity between differential calculus and discrete calculus. Analogously, there is a similarity between operators of fractional calculus and DFC.

In recent years, many scientific works have been performed with regard to fractional calculus and DFC [1,2,3,4,5,6,7]. Furthermore, Atici and Uyanik [8] studied two new monotonicity concepts for a nonnegative or nonpositive valued function defined on a discrete domain, and gave examples to illustrate connections between these new monotonicity concepts and the traditional ones. Peterson et al. [9] proved the some important results for nabla and delta fractional difference. Mozyrska and Wyrwas [10] mentioned the Caputo-, Riemann-Liouville-, and Grünwald-Letnikov-type difference initial value problems for linear fractional-order systems, and introduced the formula for the image of the discrete Mittag-Leffler matrix function in the L-transform, and also proved forms of images in the L-transform of the expressed fractional difference summation and operators. Wu and Baleanu [11] studied on the analytical aspects, and the variational iteration method is extended in a new way to solve an initial value problem. Ortigueira et al. [12] presented a derivative based discrete-time signal processing, and studied both nabla and delta derivatives, and also generalised including the fractional case. Jonnalagadda [13] discussed the dependence of solutions of nabla fractional difference equations on the initial conditions and then obtained a fractional variation of constants formula for nabla fractional difference equations involving Caputo type fractional differences. Zhou et al. [14] studied on the initial value problem to a nonlinear fractional difference equation with the Caputo like difference operator, and also obtained global and local existence results of solutions by means of some fixed point theorems. Abdeljawad and Baleanu [15] defined the right fractional sum and difference in the delta time scale calculus, and introduced a formula for the integration by parts, and then obtain a discrete Euler-Lagrange equation in fractional calculus by using this formula. An almost free damping 
vibration equation was discussed by means of $\mathrm{N}$-fractional calculus [16]. The Adomian decomposition method was applied on a fractionally damped mechanical oscillator for a sine excitation, and the analytical solution of the problem was found [17]. The solution of the fractional vibration equation, where the damping term is characterized by means of the Caputo fractional derivative was investigated [18].

The differential operators and singular differential equations that are the extensive research interests of the applied mathematics provide positive contributions for the development of the academic studies for many years [19,20,21]. In this direction, we investigated the equations of free vibration motion that can be transformed to the singular differential equations. And, we obtained the explicit solutions of these equations by applying nabla discrete fractional operator. So, we developed a different solution method for the ordinary differential equations (ODEs).

\section{Preliminaries}

In this section, we present some properties of fractional calculus and DFC.

Definition 1. There are different definitions of differentiation and integration in fractional calculus. One of these definitions is Riemann-Liouville definition. Fractional differentiation and fractional integration of this definition are given by, respectively [22],

$$
{ }_{a} D_{t}^{v} f(t)=[f(t)]_{v}=\frac{1}{\Gamma(n-v)} \frac{d^{n}}{d t^{n}} \int_{a}^{t} \frac{f(\xi)}{(t-\xi)^{v+1-n}} d \xi \quad(n-1 \leq v<n, n \in \mathbf{N}) .
$$

and,

$$
{ }_{a} D_{t}^{-v} f(t)=[f(t)]_{-v}=\frac{1}{\Gamma(v)} \int_{a}^{t} \frac{f(\xi)}{(t-\xi)^{1-v}} d \xi \quad(t>a, v>0)
$$

Definition 2. Let

$$
t^{\bar{n}}=t(t+1)(t+2) \ldots(t+n-1) \quad\left(n \in \mathbb{N}, t^{\overline{0}}=1\right),
$$

where $t^{\bar{n}}$ is the rising factorial power [23], or the ascending factorial [24]. Consider $t \in \mathbb{R} \backslash\{\ldots,-2,-1,0\}$ and $v \in \mathbb{R}$. Then " $t$ to the $v$ rising" is defined by

$$
t^{\bar{v}}=\frac{\Gamma(t+v)}{\Gamma(t)} \quad\left(0^{\bar{v}}=0\right)
$$

Then, we write equality as

$$
\nabla\left(t^{\bar{v}}\right)=v t^{\overline{v-1}}
$$

where $\nabla f(t)=f(t)-f(t-1)[5]$.

Definition 3. Let $a \in \mathbb{R}$ and $v \in \mathbb{R}^{+}$such that $0<n-1 \leq v<n \quad\left(n \in \mathbb{Z}^{+}\right)$. The $\boldsymbol{v}$-th order fractional sum of $f$ is given by

$$
\nabla_{a}^{-v} f(t)=\sum_{\xi=a}^{t} \frac{[t-h(\xi)]^{\overline{v-1}}}{\Gamma(v)} f(\xi)
$$

where $t \in \mathbb{N}_{a}=\{a, a+1, a+2, \ldots\}, h(t)=t-1$ is backward jump operator of the time scale calculus. The $v$-th order fractional difference of $f$ is given by

$$
\nabla_{a}^{v} f(t)=\nabla^{n} \nabla_{a}^{-(n-v)} f(t)=\nabla^{n} \sum_{\xi=a}^{t} \frac{[t-h(\xi)]^{\overline{n-v-1}}}{\Gamma(n-v)} f(\xi)
$$

where $f: \mathbb{N}_{a} \rightarrow \mathbb{R}[6]$. 
Definition 4. $\mathbb{E}$ shift operator is defined by

$$
\mathbb{E}^{n} f(t)=f(t+n),
$$

where $n \in \mathbb{N}[25]$.

Theorem 1. Let $v, v,>0$ and $k, l$ are scalars. So,

$$
\begin{aligned}
& \nabla^{-v} \nabla^{-v} f(t)=\nabla^{-(v+v)} f(t)=\nabla^{-v} \nabla^{-v} f(t), \\
& \nabla^{v}[k f(t)+l g(t)]=k \nabla^{v} f(t)+l \nabla^{v} g(t), \\
& \nabla \nabla^{-v} f(t)=\nabla^{-(v-1)} f(t), \\
& \nabla^{-v} \nabla f(t)=\nabla^{1-v} f(t)-\left(\begin{array}{c}
t+v-2 \\
t-1
\end{array}\right) f(0),
\end{aligned}
$$

where $f, g: \mathbb{N}_{0} \rightarrow \mathbb{R}[7]$.

Lemma 1. (Power Rule)

$$
\nabla_{a}^{-v}(t-a+1)^{\bar{v}}=\frac{\Gamma(v+1)}{\Gamma(v+v+1)}(t-a+1)^{\overline{v+v}} \quad\left(\forall t \in \mathbb{N}_{a}\right)
$$

where $v, v \in \mathbb{R}(v>0)[6]$.

Lemma 2. The following equality is hold

$$
\nabla_{a+1}^{-v} \nabla f(t)=\nabla \nabla_{a}^{-v} f(t)-\frac{(t-a+1)^{\overline{v-1}}}{\Gamma(v)} f(a) \quad(v>0)
$$

where $f$ is defined on $\mathbb{N}_{a}$ [6].

Lemma 3. (Leibniz Rule) The $v$-th order fractional difference of the product $f g$ is

$$
\nabla_{0}^{v}(f g)(t)=\sum_{n=0}^{t}\left(\begin{array}{l}
v \\
n
\end{array}\right)\left[\nabla_{0}^{v-n} f(t-n)\right]\left[\nabla^{n} g(t)\right], \quad\left(v>0, t \in \mathbb{Z}^{+}\right)
$$

where $f(t)$ and $g(t)$ are defined on $\mathbb{N}_{0}[26]$.

Lemma 4. The following equality is hold

$$
\left[f_{v}(t)\right]_{v}=f_{v+v}(t)=\left[f_{v}(t)\right]_{v} \quad\left(v, v \in \mathbb{R}, f_{v}(t) \neq 0, f_{v}(t) \neq 0\right)
$$

where $f(t)$ is analytic and single-valued function [7].

\section{Main results}

In a mass-spring system, free vibration motion of a mass is indicated by an ordinary homogeneous differential equation with arbitrary constants as follows,

$$
m \frac{d^{2} x(t)}{d t^{2}}+k x(t)=0
$$

where $x$ is amount of extension, $t$ is time, $m$ is mass and $k$ is spring constant. 


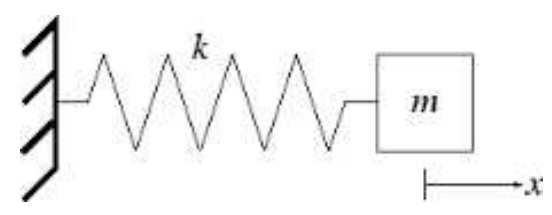

Fig. 1: Mass-spring system.

In Eq. (13), we suppose that $\mu=\sqrt{k / m}$ ( $\mu$ is vibration frequency), and so, we have

$$
\frac{d^{2} x(t)}{d t^{2}}+\mu^{2} x(t)=0
$$

This last equation can be solved simply by the help of characteristic equation. Although, usage of the nabla discrete fractional operator is not easy for Eq. (14), we want to prove existence of a different method. Firstly, we need to transform Eq. (14) to a singular differential equation due to apply the nabla operator.

Let $x=t^{2+\alpha} \varphi(t \neq 0, \varphi=\varphi(t))$. So, we write

$$
t \varphi_{2}+2(\alpha+2) \varphi_{1}+\left[\mu^{2} t+\left(\alpha^{2}+3 \alpha+2\right) t^{-1}\right] \varphi=0,
$$

where $\varphi_{n}=d^{n} \varphi / d t^{n}(n=0,1,2, \ldots)$.

For $\alpha^{2}+3 \alpha+2=0$, we obtain $\alpha=-2,-1$. In case of $\alpha=-2$, we have Eq. (14). Let $\alpha=-1$ and $\varphi=e^{\beta t} \psi(t \neq 0, \psi=\psi(t))$. Then,

$$
t \psi_{2}+2(\beta t+1) \psi_{1}+\left[\left(\beta^{2}+\mu^{2}\right) t+2 \beta\right] \psi=0 .
$$

If we choose that $\beta^{2}+\mu^{2}=0$ in Eq. (16), then, we have $\beta= \pm i \mu$, and so,

$$
t \psi_{2}+2(1 \pm i \mu t) \psi_{1} \pm 2 i \mu \psi=0
$$

By applying $\nabla$-discrete fractional operator to the both sides of (17), we have

$$
\nabla^{v}\left(t \psi_{2}\right)=t \psi_{2+v}+v \mathbb{E} \psi_{1+v}, \quad \nabla^{v}\left(t \psi_{1}\right)=t \psi_{1+v}+v \mathbb{E} \psi_{v}
$$

and,

$$
t \psi_{2+v}+[v \mathbb{E}+2(1 \pm i \mu t)] \psi_{1+v} \pm 2 i \mu(v \mathbb{E}+1) \psi_{v}=0,
$$

where $\mathbb{E}$ is the shift operator. Here, if $v \mathbb{E}+1=0, v=-\mathbb{E}^{-1}=-1$, and so, we obtain first-order homogeneous linear ordinary differential equations as

$$
\psi_{1}+\left(t^{-1}+2 i \mu\right) \psi=0, \quad \psi_{1}+\left(t^{-1}-2 i \mu\right) \psi=0
$$

Solutions of Eq. (19) are, respectively,

$$
\psi^{I}=A t^{-1} e^{-2 i \mu t}, \quad \psi^{I I}=B t^{-1} e^{2 i \mu t}
$$

where $A, B$ are arbitrary constants and, general solution of the system is

$$
x(t)=C \cos \mu t+D \sin \mu t \quad(C=A+B, D=B-A) .
$$


Definition 5. For Eq. (14), we get initial conditions as

$$
x(0)=x_{0}, \quad x_{1}(0)=v_{0},
$$

where $v_{0}$ is velocity. By using (22) for (21), we have equalities

$$
C=x_{0}, \quad D=\frac{v_{0}}{\mu} .
$$

Finally, we obtain the solution in periodic functions as follows

$$
x(t)=x_{0} \cos \mu t+\frac{v_{0}}{\mu} \sin \mu t
$$

where the motion is reciprocating action. This motion is known as simple harmonic motion.

Now, we suppose that $x(t)=E \sin (\mu t+\theta)$ in Eq. (24), that is,

$$
x(t)=E \sin \mu t \cos \theta+E \sin \theta \cos \mu t .
$$

Then, we write

$$
E \cos \theta=\frac{v_{0}}{\mu}, \quad E \sin \theta=x_{0},
$$

and,

$$
\tan \theta=\frac{\mu x_{0}}{v_{0}}, \quad E=\sqrt{\left(\frac{v_{0}}{\mu}\right)^{2}+\left(x_{0}\right)^{2}},
$$

where $\theta$ is phase angle and $E$ is vibration amplitude. Here, elapsed time for a reciprocating action is $T=2 \pi / \mu=$ $2 \pi \sqrt{m / k}$.

Example 1. Let $m=5 \mathrm{~kg}$ and $k=125 \mathrm{~N} / \mathrm{m}$. If system is pulled $x_{0}=0.4 \mathrm{~m}$ and released with $v_{0}=0.2 \mathrm{~m} / \mathrm{s}$ speed;

$$
\mu=5 \mathrm{rad} / \mathrm{s}, \quad T \cong 1.25 \mathrm{~s}, \quad x(t)=(0.4) \cos 5 t+(0.04) \sin 5 t .
$$

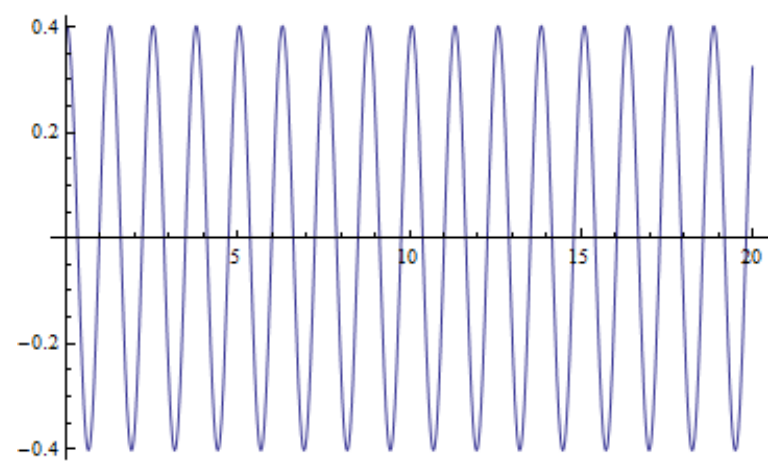

Fig. 2: The graphic of mass-spring system. 
The equation of motion in an antifriction system that is consisted by connecting a damper to previous system is

$$
x_{2} t+a x_{1} t+b x(t)=0,
$$

where $a=c / m, b=k / m$ and $c$ is damping coefficient. This equation states a mass-spring-damper system.

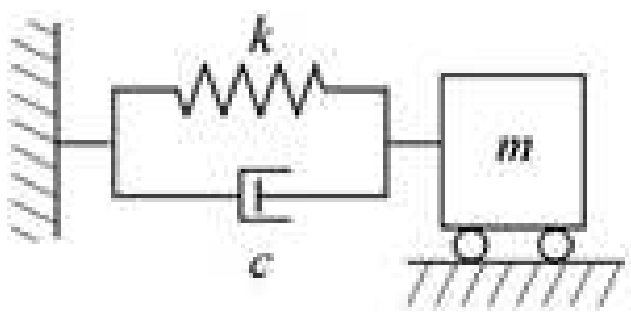

Fig. 3: Mass-spring-damper system.

By substituting $x=t^{2+\gamma} \varphi(t \neq 0, \varphi=\varphi(t))$, we obtain

$$
t \varphi_{2}+[a t+2(2+\gamma)] \varphi_{1}+\left[b t+a(2+\gamma)+\left(\gamma^{2}+3 \gamma+2\right) t^{-1}\right] \varphi=0
$$

Here, if we suppose that $\gamma=-1$ and $\varphi=e^{\delta t} \psi(t \neq 0, \psi=\psi(t))$ alike previous solution, we have

$$
t \psi_{2}+[(a+2 \delta) t+2] \psi_{1}+\left[\left(\delta^{2}+a \delta+b\right) t+a+2 \delta\right] \psi=0 .
$$

For $\delta^{2}+a \delta+b=0, \delta=\varepsilon \pm \tau$, where $\varepsilon=-a / 2$ and $\tau=\sqrt{a^{2}-4 b} / 2$ and,

$$
t \psi_{2}+(\eta t+2) \psi_{1}+\eta \psi=0
$$

where $\eta=a+2 \delta$.

By applying $\nabla$-discrete fractional operator to the both sides of (31), we have

$$
t \psi_{2+v}+(v \mathbb{E}+\eta t+2) \psi_{1+v}+\eta(v \mathbb{E}+1) \psi_{v}=0 .
$$

Here, for $v=-\mathbb{E}^{-1}=-1$,

$$
\psi_{1}+\left(t^{-1}+\eta\right) \psi=0
$$

and, solution is $\psi=\mathbf{A} t^{-1} e^{-\eta t}$ where $\mathbf{A}$ is arbitrary constant. Finally, we obtain $x(t)=\mathbf{A} e^{-\left(\frac{a+\eta}{2}\right) t}$. Here, three cases are possible,

(i) $a^{2}>4 b$ (Overdamped response). The motion will finish in a short time and, solution is $x(t)=\mathbf{B} e^{-\left(\frac{a-\sqrt{a^{2}-4 b}}{4}\right)}+\mathbf{C} e^{-\left(\frac{a+\sqrt{a^{2}+4 b}}{4}\right)}$ where $\mathbf{B}, \mathbf{C}$ are arbitrary constants.

(ii) $a^{2}=4 b$ (Criticaldamped response). This is not a vibration motion and, solution is $x(t)=(\mathbf{B}+\mathbf{C} t) e^{-\frac{a t}{4}}$.

(iii) $a^{2}<4 b$ (Underdamped response). Here, damped natural frequency $\mu_{n}$ is defined by $\mu \sqrt{1-\sigma^{2}}$, where $\sigma=c / 2 \sqrt{\mathrm{km}}$ is damping ratio and, $\mu=\sqrt{k / m}$. And so, $\sqrt{a^{2}-4 b} / 4=i \mu_{n}$ and,

$$
x(t)=e^{-\frac{a t}{4}}\left(\mathbf{B} \cos \mu_{n} t+\mathbf{C} \sin \mu_{n} t\right)
$$


Let $x(t)=\mathbf{D} e^{-\frac{a t}{4}} \cos \left(\mu_{n} t-\rho\right)$ in Eq. (34), that is,

$$
x(t)=e^{-\frac{a t}{4}}\left(\mathbf{D} \cos \mu_{n} t \cos \rho+\mathbf{D} \sin \mu_{n} t \sin \rho\right) .
$$

Then, we have

$$
\mathbf{B}^{2}+\mathbf{C}^{2}=\mathbf{D}^{2}, \quad \tan \rho=\frac{\mathbf{C}}{\mathbf{B}}
$$

where $e^{-\frac{a t}{4}}$ is damping factor and, the motion is a vibration motion.

Example 2. Let $m=5 \mathrm{~kg}, c=40 \mathrm{~kg} / \mathrm{s}$ and $k=100 \mathrm{~N} / \mathrm{m}$ in a mass-spring-damper system. And, initial conditions are $x(0)=0, x_{1}(0)=0.4 \mathrm{~m} / \mathrm{s}$. Then, we obtain

$$
a=8, \quad \sigma \cong 0.89<1, \quad \mu \cong 4.47 s^{-1}, \quad \mu_{n} \cong 2.01 s^{-1}
$$

and,

$$
x(t)=e^{-2 t}[\mathbf{B} \cos (2.01) t+\mathbf{C} \sin (2.01) t] .
$$

Under the initial conditions, the solution is

$$
x(t)=(0.4) e^{-2 t} \sin (2.01) t
$$

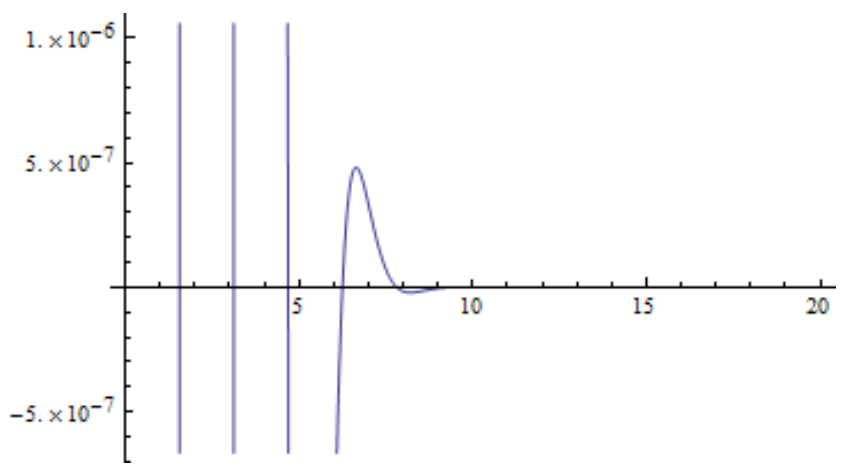

Fig. 4: The graphic of mass-spring-damper system.

\section{Conclusion}

We investigated the equations of free vibration motion in a mass-spring system and mass-spring-damper system. And, we obtained the solutions by the help of the nabla discrete fractional operator. After, this paper was enriched by means of some examples. And, we will apply this method to the another singular equations in the future time.

\section{Acknowledgment}

The author would like to thanks the referees for useful and improving comments.

\section{Competing Interests}

The authors declare that they have no competing interests. 


\section{Authors' Contributions}

All authors have contributed to all parts of the article. All authors read and approved the final manuscript.

\section{References}

[1] K.B. Oldham and J. Spanier, The Fractional Calculus, Academic Press Cambridge, MA, USA (1974).

[2] J.B. Diaz and T.J. Osler, Differences of fractional order, American Mathematical Society, 28, (1974), 185-202.

[3] K.S. Miller and B. Ross, An Introduction to the Fractional Calculus and Fractional Differential Equation, 1st ed., Wiley, NJ, USA, (1993).

[4] I. Podlubny, Matrix approach to discrete fractional calculus, Fract Calc Appl Anal., 3 (4), (2000), 359-386.

[5] F.M. Atici and P.W. Eloe, Discrete fractional calculus with the nabla operator, Electron. J. Qual. Theory. Differ. Equ., 3, (2009), $1-12$.

[6] F.M. Atici and N. Acar, Exponential functions of discrete fractional calculus, Appl. Anal. Discrete. Math., 7, (2013), $343-353$.

[7] R. Yilmazer, M. Inc, F. Tchier and D. Baleanu, Particular solutions of the confluent hypergeometric differential equation by using the nabla fractional calculus operator, Entropy, 18 (2), (2016), 49.

[8] F.M. Atici and M. Uyanik, Analysis of discrete fractional operators, Appl. Anal. Discrete Math., 9 (1), (2015), 139-149.

[9] J. Baoguo, L. Erbe and A. Peterson, Convexity for nabla and delta fractional differences, Journal of Difference Equations and Applications, 21 (4), (2015), 360-373.

[10] D. Mozyrska and M. Wyrwas, The L-transform method and delta type fractional difference operators, Discrete Dynamics in Nature and Society, 2015, (2015), 12 pages.

[11] G.-C. Wu and D. Baleanu, New applications of the variational iteration method-from differential Equations to q-fractional difference equations, Advances in Difference Equations, 2013 (21), (2013), 16 pages.

[12] M.D. Ortigueira, F.J.V. Coito and J.J. Trujillo, A new look into the discrete-time fractional calculus: Derivatives and exponentials, fractional differentiation and its applications, 6 (1), (2013), 629-634.

[13] J. Jonnalagadda, Solutions of perturbed linear nabla fractional difference equations, Differ. Equ. Dyn. Syst., 22 (3), (2014) $281-292$.

[14] F. Chen, X. Luo and Y. Zhou, Existence results for nonlinear fractional difference equation, Advances in Difference Equations, 2011, (2011), 12 pages.

[15] T. Abdeljawad and D. Baleanu, Fractional differences and integration by parts, Journal of Computational Analysis and Applications, 13 (3), (2011), 574-582.

[16] T. Miyakoda, On an almost free damping vibration equation using N-fractional calculus, Journal of Computational and Applied Mathematics, 144, (2002), 233-240.

[17] A. Palfalvi, Efficient solution of a vibration equation involving fractional derivatives, International Journal of Non-Linear Mechanics, 45, (2010), 169-175.

[18] L.-L. Liu and J.-S. Duan, A detailed analysis for the fundamental solution of fractional vibration equation, Open Mathematics, 13 (1), (2015), 2391-5455.

[19] E.S. Panakhov and M. Sat, Inverse problem for the interior spectral data of the equation of hydrogen atom, Ukrainian Mathematical Journal, 64 (11), (2013), 1716-1726.

[20] M. Sat and E.S. Panakhov, A uniqueness theorem for Bessel operator from interior spectral data, Abstr Appl Anal., 2013, (2013), 6 pages.

[21] M. Sat and E.S. Panakhov, Spectral problem for diffusion operator, Applicable Analysis, 93 (6), (2014), 1178-1186.

[22] R. Yilmazer and O. Ozturk, Explicit solutions of singular differential equation by means of fractional calculus operators, Abstr Appl Anal., 2013, (2013), 6 pages.

[23] R.L. Graham, D.E. Knuth and O. Patashnik, Concrete Mathematics: A Foundation for Computer Science, 2nd ed., Addison-Wesley, Reading, MA, USA, (1994).

[24] G. Boros and V. Moll, Irresistible Integrals: Symbols, Analysis and Experiments in the Evaluation of Integrals, Cambridge University Press, Cambridge, (2004).

[25] W.G. Kelley and A.C. Peterson, Difference Equations: An Introduction with Applications. Academic Press, Cambridge, MA, USA, (2001).

[26] J.J. Mohan and G.V.S.R. Deekshitulu, Solutions of fractional difference equations using S-transforms, Malaya J Math., 3 (1), (2013), 7-13. 